Document downloaded from:

http://hdl.handle.net/10251/158397

This paper must be cited as:

Bermudez, T.; Bonilla, A.; Müller, V.; Peris Manguillot, A. (2019). Ergodic and dynamical properties of m-isometries. Linear Algebra and its Applications. 561:98-112. https://doi.org/10.1016/j.laa.2018.09.022

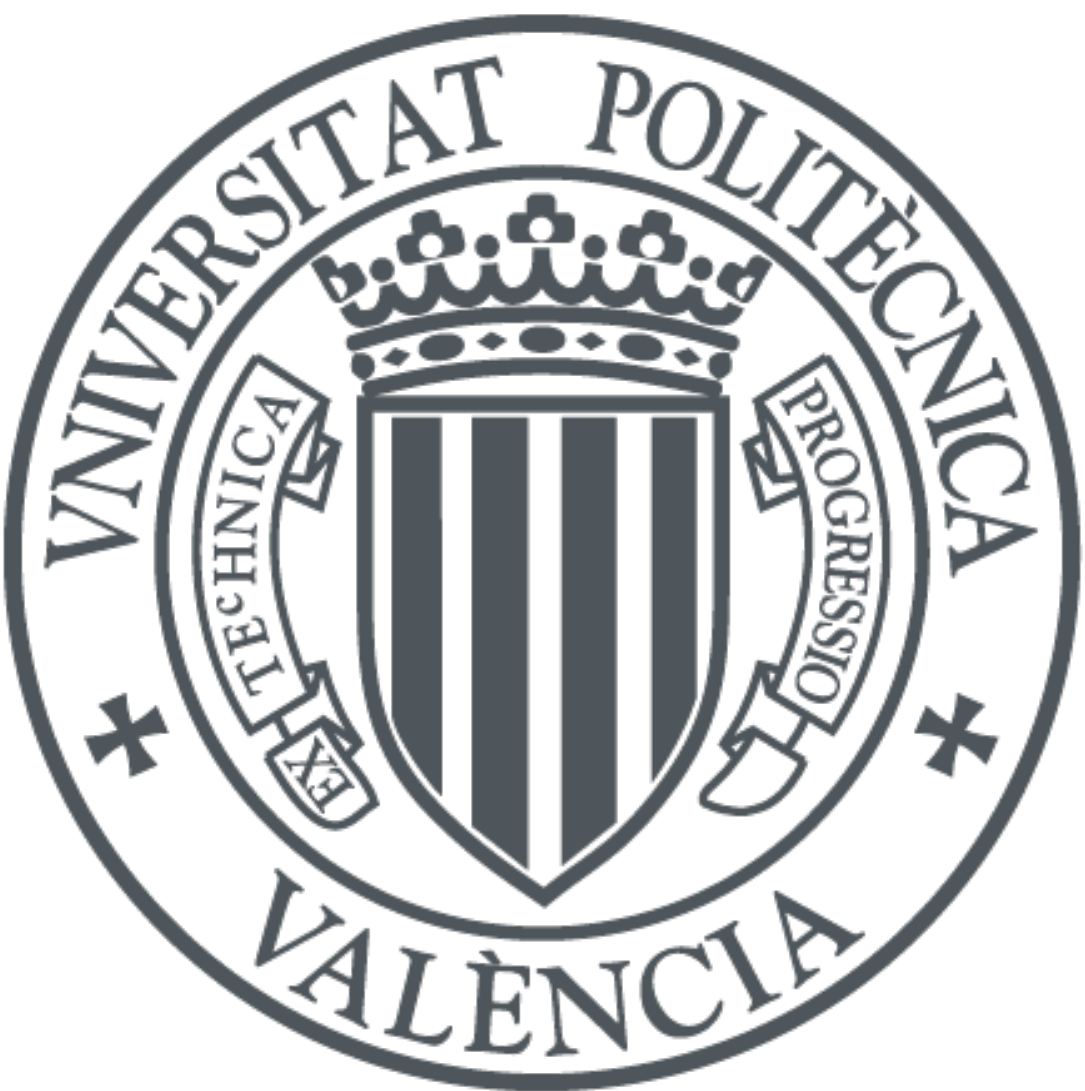

The final publication is available at

https://doi.org/10.1016/j.laa.2018.09.022

Copyright Elsevier

Additional Information 


\title{
Ergodic and dynamical properties of $m$-isometries
}

\author{
T. Bermúdez, A. Bonilla, V. Müller and A. Peris *
}

March 14, 2018

\begin{abstract}
An example of a weakly ergodic 3 -isometry is provided in [3], we give new examples of weakly ergodic 3-isometries and study numerically hypercyclic $m$ isometries on finite and infinite dimensional Hilbert spaces. In particular, all weakly ergodic strict 3-isometries on a Hilbert space are weakly numerically hypercyclic. Adjoints of unilateral forward weighted shifts which are strict $m$-isometries on $\ell^{2}(\mathbb{N})$ are shown to be hypercyclic.
\end{abstract}

\section{Introduction}

Throughout this article $X$ stands for a Banach space, the symbol $B(X)$ denotes the space of bounded linear operators defined on $X$.

Given $T \in B(X)$, we denote the Cesàro mean by

$$
M_{n}(T) x:=\frac{1}{n+1} \sum_{k=0}^{n} T^{k} x \text { for all } x \in X .
$$

We need to recall some definitions concerning the behaviour of the sequence of Cesàro means $\left(M_{n}(T)\right)_{n \in \mathbb{N}}$.

Definition 1.1. A linear operator $T$ on a Banach space $X$ is called

1. Uniformly ergodic if $M_{n}(T)$ converges uniformly.

2. Mean ergodic if $M_{n}(T)$ converges in the strong operator topology of $X$.

3. Weakly ergodic if $M_{n}(T)$ converges in the weak operator topology of $X$.

4. Absolutely Cesàro bounded if there exists a constant $C>0$ such that

$$
\sup _{N \in \mathbb{N}} \frac{1}{N} \sum_{j=1}^{N}\left\|T^{j} x\right\| \leq C\|x\| \quad \text { for all } x \in X .
$$

*The first, second and fourth authors were supported by MINECO and FEDER, Project MTM2016-75963-P. The third author was supported by grant No. 17-27844S of GA CR and RVO: 67985840. The fourth author was also supported by Generalitat Valenciana, Project PROMETEO/2017/102. 
5. Cesàro bounded if the sequence $\left(M_{n}(T)\right)_{n \in \mathbb{N}}$ is bounded.

6. Uniformly Kreiss bounded if there is a $C>0$ such that

$$
\left\|M_{n}(\lambda T)\right\| \leq C \text { for }|\lambda|=1 \text { and } n=0,1,2, \cdots .
$$

An operator $T$ is said power bounded if there is a $C>0$ such that $\left\|T^{n}\right\|<C$ for all $n$.

Remark 1.1. 1. On finite-dimensional Hilbert spaces, the classes of uniformly Kreiss bounded and power bounded operators are equal.

2. Any absolutely Cesàro bounded operator is uniformly Kreiss bounded, by (1).

The class of absolutely Cesàro bounded operators was introduced by Hou and Luo in [16].

The following implications for operators on Hilbert spaces among various concepts in ergodic theory are a direct consequence of the corresponding definitions and results in $[5]$ :

$$
\begin{aligned}
& \left\|\frac{T^{n}}{\sqrt{n}}\right\| \rightarrow 0 \quad\left\|\frac{T^{n}}{n}\right\| \rightarrow 0 \\
& \uparrow \quad \uparrow
\end{aligned}
$$

Power bounded $\Longrightarrow$ Abs. Cesàro bounded $\Longrightarrow$ Uniformly Kreiss bounded

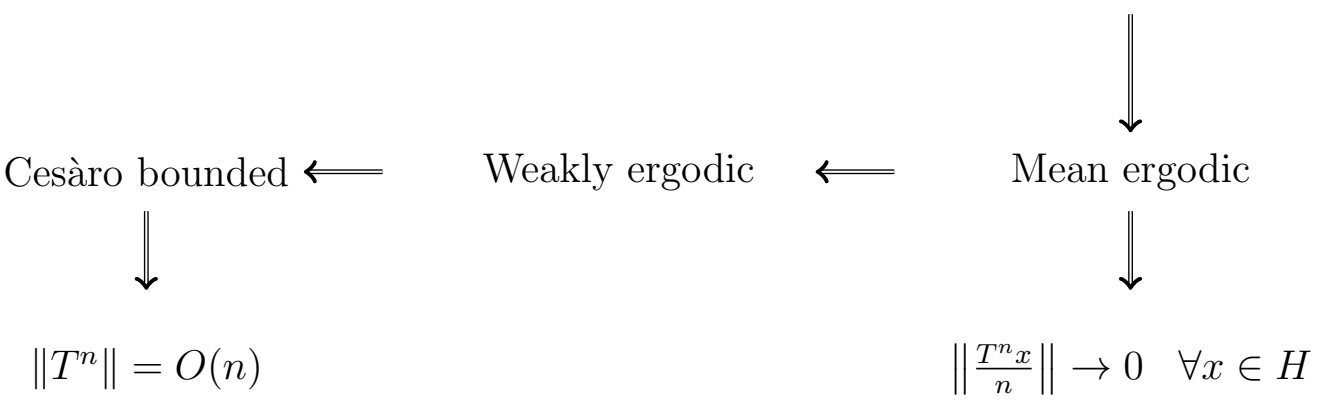

Figure 1: Relations between different definitions in ergodic theory in Hilbert spaces.

In general, the converse implications of the above figure are not true.

Let $H$ be a Hilbert space. For a positive integer $m$, an operator $T \in B(H)$ is called an m-isometry if for any $x \in H$,

$$
\sum_{k=0}^{m}(-1)^{m-k}\left(\begin{array}{c}
m \\
k
\end{array}\right)\left\|T^{k} x\right\|^{2}=0 .
$$

We say that $T$ is a strict m-isometry if $T$ is an $m$-isometry but it is not an $(m-1)$-isometry. 
Remark 1.2. 1. For $m \geq 2$, the strict $m$-isometries are not power bounded. Moreover, $\left\|T^{n}\right\|=O(n)$ for 3 -isometries and $\left\|T^{n}\right\|=O\left(n^{\frac{1}{2}}\right)$ for 2-isometries.

2. There are no strict $m$-isometries on finite dimensional spaces for $m$ even. See [2, Proposition 1.23].

3. An example of a weakly ergodic 3 -isometry is provided in [3].

We recall the following definition that allow us to study some properties of orbits of the $m$-isometries or adjoint of $m$-isometries.

An operator $T \in B(X)$ is said to be hypercyclic if there exists a point $x \in X$ such that for every nonempty open subset $U$ of $X$, the set $\left\{n \in \mathbb{N}: T^{n} x \in U\right\}$ is nonempty, $T$ is mixing if for every nonempty open sets $U, V \subset X$, there exists $n_{0} \in \mathbb{N}$ such that $T^{n}(U) \cap V \neq \emptyset$ for all $n \geq n_{0}$ and $T$ is Devaney chaotic if it is hypercyclic and has a dense set of periodic points.

Examples of absolutely Cesàro bounded mixing operators on $\ell^{p}(\mathbb{N})$ are given in [5] (see also [16], [9], [10]).

Definition 1.2. Let $H$ be a Hilbert space. $T \in B(H)$ is called numerically hypercyclic if there exists a unit vector $x \in H$ such that the set $\left\{\left\langle T^{n} x, x\right\rangle: n \in \mathbb{N}\right\}$ is dense in $\mathbb{C}$.

Clearly numerical hypercyclicity is preserved by unitary equivalence but in general not by similarity. This leads to the following definition:

Definition 1.3. Let $T \in B(X)$. It is said that $T$ is weakly numerically hypercyclic if $T$ is similar to a numerically hypercyclic operator.

In [21, Proposition 1.5], Shkarin proved that $T \in B(H)$ is weakly numerically hypercyclic if and only if there exist $x, y \in H$ such that the set $\left\{\left\langle T^{n} x, y\right\rangle: n \in \mathbb{N}\right\}$ is dense in $\mathbb{C}$.

The paper is organized as follows: Section 2 studies ergodic properties of $m$ isometries on finite or infinite dimensional Hilbert spaces. For example, strict $m$ isometries with $m>3$ are not Cesàro bounded, and we give new examples of weakly ergodic 3-isometries. In Section 3, we analyze numerical hypercyclicity of $m$-isometries. In particular, we obtain that the adjoint of any strict $m$-isometry unilateral forward weighted shift on $\ell^{2}(\mathbb{N})$ is hypercyclic. Moreover, we prove that weakly ergodic 3-isometries are weakly numerically hypercyclic.

\section{Ergodic properties for $m$-isometries in Hilbert spaces}

The purpose of this section is to study ergodic properties of $m$-isometries. It is clear that isometries (1-isometries) are power bounded. It is natural to ask about strict $m$-isometries and the definitions of Figure 1 on finite or infinite Hilbert spaces. 
The following example is due to Assani. See [13, page 10] and [3, Theorem 5.4] for more details.

Example 2.1. Let $H$ be $\mathbb{R}^{2}$ or $\mathbb{C}^{2}$ and $T=\left(\begin{array}{cc}-1 & 2 \\ 0 & -1\end{array}\right)$. It is clear that

$$
T^{n}=\left(\begin{array}{cc}
(-1)^{n} & (-1)^{n-1} 2 n \\
0 & (-1)^{n}
\end{array}\right)
$$

and $\sup _{n \in \mathbb{N}}\left\|M_{n}(T)\right\|<\infty$. Then $T$ is Cesàro bounded and $\frac{\left\|T^{n} x\right\|}{n}$ does not converge to 0 for some $x \in H$. Hence $T$ is not mean ergodic. Note that $T$ is a strict 3 -isometry.

The above example shows that on a 2-dimensional Hilbert space there exists a 3 -isometry which is Cesàro bounded and not mean ergodic. This example could be generalized to any Hilbert space of dimension greater or equal to 2.

Let $H$ be a Hilbert space and $T \in B(H)$. Tomilov and Zemánek in [22] considered the Hilbert space $\mathcal{H}=H \oplus H$ with the norm

$$
\left\|x_{1} \oplus x_{2}\right\|_{H \oplus H}=\sqrt{\left\|x_{1}\right\|^{2}+\left\|x_{2}\right\|^{2}},
$$

and the bounded linear operator $\mathcal{T}$ on $\mathcal{H}$ given by the matrix

$$
\mathcal{T}:=\left(\begin{array}{cc}
T & T-I \\
0 & T
\end{array}\right)
$$

In fact, they obtained the following relations of ergodic properties between the operators $\mathcal{T}$ and $T$.

Lemma 2.1. [22, Lemmma 2.1] Let $T \in B(H)$. Then

1. $\mathcal{T}$ is Cesàro bounded if and only if $T$ is power bounded.

2. $\mathcal{T}$ is mean ergodic if and only if $T^{n}$ converges in the strong topology of $H$.

3. $\mathcal{T}$ is weakly ergodic if and only if $T^{n}$ converges in the weak topology of $H$.

Recall some properties of $m$-isometries.

Lemma 2.2. Let $T \in B(H)$ and $m \in \mathbb{N}$. Then

1. [7, Theorem 2.1] $T$ is a strict $m$-isometry if and only if $\left\|T^{n} x\right\|^{2}$ is the value at $n$ of a polynomial of degree less or equal to $m-1$ for all $x \in H$, and there exists $x_{m} \in H$ such that $\left\|T^{n} x_{m}\right\|^{2}$ is a polynomial of degree exactly $m-1$.

2. [8, Theorem 2.7] If $H$ is a finite dimensional Hilbert space, then $T$ is a strict $m$-isometry with odd $m$ if and only if there exist a unitary $U \in B(H)$ and a nilpotent operator $Q \in B(H)$ of order $\frac{m+1}{2}$ such that $U Q=Q U$ with $T=U+Q$. 
3. [8, Theorem 2.2] If $A \in B(H)$ is an isometry and $Q \in B(H)$ is a nilpotent operator of order $n$ which commutes with $A$, then $A+Q$ is a strict $(2 n-1)$ isometry.

Example 2.2. Let $H$ be a Hilbert space and $T \in B(H)$ such that $T=I+Q$ where $Q^{n}=0$ for some $n \geq 2$ and $Q^{n-1} \neq 0$. Define the Hilbert space $\mathcal{H}$ and the bounded linear operator $\mathcal{T}$ on $\mathcal{H}$ as above. By construction $\mathcal{T}=A+\mathcal{Q}$ where

$$
A:=\left(\begin{array}{cc}
I & 0 \\
0 & I
\end{array}\right), \quad \mathcal{Q}:=\left(\begin{array}{cc}
Q & Q \\
0 & Q
\end{array}\right)
$$

where $\mathcal{Q}^{n}=0$ and $\mathcal{Q}^{n-1} \neq 0$. By part (3) of Lemma $2.2, T$ is a strict $(2 n-1)$ isometry and hence not power bounded. Thus, by Lemma 2.1 we have that $\mathcal{T}$ is not Cesàro bounded. Newly by part (3) of Lemma $2.2, \mathcal{T}$ is a strict $(2 n-1)$-isometry.

Example 2.3. Let $\lambda$ be a unimodular complex number different from 1 . Then

$$
\mathcal{T}:=\left(\begin{array}{cc}
\lambda & \lambda-1 \\
0 & \lambda
\end{array}\right)
$$

is a Cesàro bounded operator on $\mathbb{C}^{2}$ by Lemma $2.1\left(\right.$ since $\left.\sup _{n}\left|\lambda^{n}\right|<\infty\right)$, it is not mean ergodic (since $\lambda^{n} x$ does not converge) and by Lemma $2.2, \mathcal{T}$ is a 3 -isometry on $\mathbb{C}^{2}$.

Now we give some ergodic properties of $m$-isometries.

Example 2.1 is a Cesàro bounded 3-isometry. However, as a consequence of $[5$, Theorem 2.2] and Lemma 2.2, we obtain the following.

Corollary 2.1. There is no uniformly Kreiss bounded strict 3-isometry.

Theorem 2.1. Assume that $H$ is a finite $n$-dimensional Hilbert space. Then

1. If $n \geq 2$, then there exists a Cesáro bounded strict 3-isometry.

2. The isometries are the only mean ergodic strict $m$-isometries on $H$.

Proof. (1) Let

$$
\mathcal{A}:=\left(\begin{array}{cc}
\lambda & \lambda-1 \\
0 & \lambda
\end{array}\right)
$$

be the operator on $\mathbb{C}^{2}$ considered in Example 2.3. Write $H=\mathbb{C}^{2} \oplus \mathbb{C}^{n-2}$ and let $\mathcal{B}:=\mathcal{A} \oplus I_{\mathbb{C}^{n-2}}$. Then $\mathcal{B}$ is a strict 3 -isometry which is Cesàro bounded (and not power bounded).

(2) Suppose that $T$ is a strict $m$-isometry with $m>1$ on a finite dimensional Hilbert space, then $m \geq 3$. Using part (1) of Lemma 2.2, it is easy to prove that $\frac{\left\|T^{n} x\right\|}{n}$ does not converges to 0 for some $x \in H$. So, $T$ is not mean ergodic.

In Hilbert space of infinite dimensional we can say more.

Theorem 2.2. Let $T$ be a strict m-isometry. Then 
1. If $m>3$, then $T$ is not Cesàro bounded.

2. If $m \geq 3$, then $T$ is not mean ergodic.

Proof. By part (1) of Lemma 2.2, there exists $x \in H$ such that $\left\|T^{n} x\right\|^{2}$ is a polynomial at $n$ of order $m-1$ exactly. Since

$$
\frac{T^{n}}{n+1}=M_{n}(T)-\frac{n}{n+1} M_{n-1}(T)
$$

the proof is complete.

Since any weakly ergodic operator is Cesàro bounded, in particular there is no weakly ergodic strict $m$-isometry for $m>3$.

Theorem 2.3. There exists a Cesàro bounded and weakly ergodic strict 3-isometry.

Proof. Let $U$ be the bilateral shift. Define

$$
\mathcal{M}:=\left(\begin{array}{cc}
U & U-I \\
0 & U
\end{array}\right) .
$$

First observe that $\mathcal{M}$ is Cesàro bounded, by part (1) of Lemma 2.1. Since $U^{n} \rightarrow 0$ in the weak operator topology, $\mathcal{M}$ is weakly ergodic by part (3) of Lemma 2.1 . Therefore, the conclusion is derived by part (3) of Lemma 2.2.

In [3, Section 5.2], it is given an example of Cesàro bounded strict 3-isometry $T$ on a Hilbert space $H$ for which the sequence $\left(\frac{T^{n}}{n}\right)_{n \in \mathbb{N}}$ is bounded below for all $x \in H \backslash\{0\}$. In particular, $\left(M_{n}(T) x\right)_{n \in \mathbb{N}}$ diverges for each $x \in H \backslash\{0\}$, and $T$ is weakly ergodic.

We give a characterization of this property.

Given an $m$-isometry $T$, the covariance operator of $T$ is defined by

$$
\Delta_{T}:=\frac{1}{(m-1) !} \sum_{j=0}^{m-1}(-1)^{m-1-j}\left(\begin{array}{c}
m-1 \\
j
\end{array}\right) T^{* j} T^{j} .
$$

Theorem 2.4. Let $T$ be a strict 3-isometry on a Hilbert space $H$. Then the sequence $\left(\frac{T^{n} x}{n}\right)_{n \in \mathbb{N}}$ is bounded below for all $x \in H \backslash\{0\}$ if and only if the covariance operator $\Delta_{T}$ is injective.

Proof. If $T$ is a strict 3 -isometry and $\Delta_{T}$ is injective, then $\inf _{n} \frac{\left\|T^{n} x\right\|}{n}>0$ for all $x \in H \backslash\{0\}$ (see the proof of [6, Theorem 3.4]).

If $\Delta_{T}$ is not injective, then there exists $x$ such that $\left\langle\Delta_{T} x, x\right\rangle=0$. By [6, Proposition 2.3], we have that $\inf _{n} \frac{\left\|T^{n} x\right\|}{n} \rightarrow\left\langle\Delta_{T} x, x\right\rangle=0$, and thus the sequence $\frac{T^{n} x}{n}$ is not bounded below. 
There exist weakly ergodic strict 3-isometries with the covariance operator $\Delta_{T}$ injective by [3, Section 5.2] and not injective, see the proof of Theorem 2.3.

The Uniform ergodic theorem of Lin [18, Theorem] asserts that if $\frac{\left\|T^{n}\right\|}{n} \rightarrow 0$, then $T$ is uniformly ergodic if and only if the range of $I-T$ is closed. On the other hand, $T$ is uniformly ergodic if and only if $\frac{\left\|T^{n}\right\|}{n} \rightarrow 0$ and 1 is a pole of the resolvent operator, [12].

Corollary 2.2. For $m>1$, there is no uniform ergodic strict $m$-isometry on a Hilbert space.

Proof. By part (2) of Theorem 2.2, there is no mean ergodic strict $m$-isometry for $m \geq 3$. For $m=2$ the result follows from the fact that the spectrum of any strict 2-isometry is $\sigma(T)=\overline{\mathbb{D}}$ and, thus, 1 is not an isolated point of $\sigma(T)$.

There exists a strict 3-isometry $T$ which is weakly ergodic (thus Cesàro bounded), but it is not mean ergodic. For 2-isometries something else can be established.

Corollary 2.3. Let $H$ be an infinite dimensional Hilbert space and let $T$ be a strict 2-isometry. Then the following assertions are equivalent:

1. $T$ is mean ergodic.

2. $T$ is weakly ergodic.

3. $T$ is Cesàro bounded.

Proof. It is a consequence of part (1) of Lemma 2.2, since $\frac{T^{n} x}{n}$ converges to zero for all $x \in H$.

The following example provides a 2-isometry that is not Cesàro bounded.

Example 2.4. On $\ell^{2}(\mathbb{N})$ we consider the operator $T$ given by $T\left(x_{1}, x_{2}, \ldots\right):=$ $\left(x_{1}, x_{1}, x_{2}, x_{3}, \ldots\right)$. Then $T$ is a 2-isometry which is not Cesàro bounded.

Proposition 2.1. There is no Cesàro bounded weighted forward shift on $\ell^{2}(\mathbb{N})$, which is a strict 2-isometry.

Proof. Assume that $T$ is a weighted forward shift with weights $\left(w_{n}\right)_{n \in \mathbb{N}}$. By $[1$, Theorem 1] (see also [7, Remark 3.9]), if $T$ is a strict 2-isometry, then

$$
\left|w_{n}\right|^{2}=\frac{p(n+1)}{p(n)}
$$

where $p$ is a polynomial of degree 1 , that is, $p(n):=a n+b$.

First, suppose that $b=0$. Then $w_{n}=\sqrt{\frac{n}{n-1}}$, since $a \neq 0$. Hence $T^{*} e_{n}:=$ $\sqrt{\frac{n}{n-1}} e_{n-1}$. By [5, Proposition 2.1], $T^{*}$ is not Cesàro bounded. Since Cesàro boundedness is preserved by taking adjoints, $T$ is not Cesàro bounded. 
Now, assume that $b \neq 0$, then $w_{n}(c):=\sqrt{\frac{c n+1}{c(n-1)+1}}$ with $c \neq 0$. Denote $T_{c} e_{n}:=$ $w_{n}(c) e_{n+1}$ and the diagonal operator $V e_{n}:=\alpha_{n} e_{n}$, where $\alpha_{n}:=\sqrt{\frac{c(n-1)+1}{n}}$. Then $V$ is invertible and satisfies that $V T_{1}=V T_{c}$. Moreover, $T_{1}$ is not Cesàro bounded, by following an argument as in [5, Proposition 2.1]. Using that Cesàro boundedness is preserved by similarities, we obtain that $T_{c}$ is not Cesàro bounded.

Corollary 2.4. There is no absolutely Cesàro bounded strict 2-isometry on a Hilbert space.

Proof. It is immediate by [5, Theorem 2.5] and part (1) of Lemma 2.2.

Question 2.1. Is it possible to construct a Cesàro bounded strict 2-isometry on an infinite dimensional Hilbert space?

\section{$3 \quad$ Numerically hypercyclic properties of $m$-isometries}

In this section we study numerically hypercyclic $m$-isometries. For simplicity we discuss only operators on Hilbert spaces.

Faghih and Hedayatian proved in [14] that $m$-isometries on a Hilbert space are not weakly hypercyclic. Moreover, $m$-isometries on a Banach space are not 1-weakly hypercyclic [4]. However, there are isometries that are weakly supercyclic [20] (in particular cyclic). Thus the first natural question is the following: are there numerically hypercyclic $m$-isometries?

Let $H$ be a Hilbert space. Denote

$$
I_{m}(H):=\{T \in B(H) \quad: \quad T \text { is } m \text {-isometry }\} .
$$

If $H$ is an $n$ dimensional Hilbert space, then by [8, Theorem 2.7] we have that

$I_{1}(H)=I_{2}(H) \subset I_{3}(H)=I_{4}(H) \subset \cdots \subset I_{2 n-3}(H)=I_{2 n-2}(H) \subset I_{2 n-1}(H)=I_{m}(H)$

for all $m \geq 2 n-1$.

Theorem 3.1. There are no weakly numerically hypercyclic $m$-isometries in $B\left(\mathbb{C}^{n}\right)$ for $n \leq 3$.

Proof. If $n=1$, there are not weakly numerically hypercyclic operators. Let $n=2$. By [21, Theorem 1.13], if $T \in B\left(\mathbb{C}^{2}\right)$ is a weakly numerically hypercyclic operator, then there exists $\lambda \in \sigma(T)$, with $|\lambda|>1$ and thus $T$ is not an $m$-isometry. For $n=3$, it is the same by [21, Theorem 1.14].

We discuss the existence of weakly numerically hypercyclic $m$-isometries on $n$ dimensional spaces for $n \geq 4$.

We say that $\lambda_{1}, \lambda_{2} \in \mathbb{T}$ are rationally independent if $\lambda_{1}^{m_{1}} \lambda_{2}^{m_{2}} \neq 1$ for every nonzero pair $m=\left(m_{1}, m_{2}\right) \in \mathbb{Z}^{2}$, or equivalently if $\lambda_{j}=e^{i \theta_{j}}$ with $\theta_{j} \in \mathbb{R}$ with $\pi, \theta_{1}, \theta_{2}$ are linearly independent over the field $\mathbb{Q}$ of rational numbers. 
If $T \in B(X)$ and there are rationally independent $\lambda_{1}, \lambda_{2} \in \mathbb{T}$ such that $\operatorname{ker}(T-$ $\left.\lambda_{j} I\right)^{2} \neq \operatorname{ker}\left(T-\lambda_{j} I\right)$ for $j \in\{1,2\}$, then $T$ is weakly numerically hypercyclic [21, Theorem 1.9]. Moreover if $X$ is a Hilbert space, then $T$ is numerically hypercyclic [21, Proposition 1.12]. The following result gives an answer to the above question for some $m$-isometries.

Theorem 3.2. There exists a numerically hypercyclic strict $(2 m-1)$-isometry on $B\left(\mathbb{C}^{n}\right)$, with $n \geq 4$, for $2 \leq m \leq n-2$.

Proof. Let $\ell \in\{2,3, \ldots, n-2\}$. We will construct a numerically hypercyclic strict $(2 \ell-1)$-isometry. Define $D$ the diagonal operator with diagonal

$$
(\underbrace{\lambda_{1}, \cdots, \lambda_{1}}_{\ell}, \lambda_{2}, \lambda_{2}, \underbrace{1, \cdots, 1}_{k-2 \ell})
$$

where $\lambda_{1}$ and $\lambda_{2}$ are rationally independent complex numbers with modulus 1 and $Q$ by

$$
\begin{aligned}
Q e_{i}: & =e_{i-1} \text { for } i \in\{2,3, \cdots, \ell\} \\
Q e_{\ell+2}: & =e_{\ell+1} \text { and } \\
Q e_{i}: & =0 \text { for } i=1, i=\ell+1 \text { and } i \geq \ell+3 .
\end{aligned}
$$

It is clear that $Q^{\ell}=0$ and $Q^{\ell-1} e_{\ell}=e_{1} \neq 0$. Moreover,

$$
\begin{aligned}
Q D e_{i} & =D Q e_{i}=\lambda_{1} e_{i-1} \text { for } 2 \leq i \leq \ell \\
Q D e_{\ell+2} & =D Q e_{\ell+2}=\lambda_{2} e_{\ell+1} \\
Q D e_{i} & =D Q e_{i}=0 \text { for } i=1, \ell+1 \text { and } \geq i \geq \ell+3 .
\end{aligned}
$$

By part (3) of Lemma 2.2, $T:=D+Q$ is a strict $(2 \ell-1)$-isometry for any $\ell \in$ $\{2,3, \cdots, n-2\}$.

Let us prove that $T$ satisfies that $\operatorname{Ker}\left(\lambda_{i}-T\right) \neq \operatorname{Ker}\left(\lambda_{i}-T\right)^{2}$ for $i=1,2$. By definition $e_{2} \in \operatorname{Ker}\left(\lambda_{1}-T\right)^{2} \backslash \operatorname{Ker}\left(\lambda_{1}-T\right)$ and $e_{\ell+1} \in \operatorname{Ker}\left(\lambda_{2}-T\right)^{2} \backslash \operatorname{Ker}\left(\lambda_{2}-T\right)$. So by [21, Proposition 1.9], $T$ is numerically hypercyclic.

As a consequence of the proof of Theorem 3.2, we obtain

Corollary 3.1. Let $H$ be a complex Hilbert space with dimension at least 4. Then there exists a numerically hypercyclic strict 3-isometry on $H$.

Theorem 3.3. An n-dimensional Hilbert space supports no weakly numerically hypercyclic strict $(2 n-3)$ or $(2 n-1)$-isometries.

Proof. Let $H$ be a finite-dimensional Hilbert space, $\operatorname{dim} H=n<\infty$. Suppose on the contrary that $T \in B(H)$ is a weakly numerically hypercyclic $(2 n-1)$-isometry. Since $\left\|T^{k} x\right\|^{2}$ grows polynomially for each $x \in H$ and there exists $u \in H$ such that $\left\|T^{k} u\right\|^{2}$ is a polynomial of degree $2 n-2$, the Jordan form of $T$ has only one block 
corresponding to an eigenvalue $\lambda$ with $|\lambda|=1$. Thus $T=\lambda I+Q$ where $Q^{n}=0$. Thus

$$
T^{k}=\sum_{j=0}^{k}\left(\begin{array}{c}
k \\
j
\end{array}\right) \lambda^{k-j} Q^{j}=\lambda^{k} \sum_{j=0}^{k}\left(\begin{array}{l}
k \\
j
\end{array}\right) \lambda^{-j} Q^{j}
$$

for all $k \in \mathbb{N}$.

Let $x, y \in H$ and suppose that the set $\left\{\left\langle T^{k} x, y\right\rangle: k \in \mathbb{N}\right\}$ is dense in $\mathbb{C}$. We have $\left\langle T^{k} x, y\right\rangle=\lambda^{k} p(k)$ for some polynomial $p$ of degree $\leq n-1$. If $\operatorname{deg} p \geq 1$ then $\left|\left\langle T^{k} x, y\right\rangle\right| \rightarrow \infty$ so the set $\left\{\left\langle T^{k} x, y\right\rangle: k \in \mathbb{N}\right\}$ is not dense in $\mathbb{C}$.

If $\operatorname{deg} p=0$ then the set $\left\{\left\langle T^{k} x, y\right\rangle: k \in \mathbb{N}\right\}$ is bounded and again is not dense in $\mathbb{C}$. Hence $T$ is not weakly numerically hypercyclic.

The case of $(2 n-3)$-isometries can be treated similarly. If $T \in B(H)$ is a strict $(2 n-3)$-isometry then the Jordan form of $T$ has two blocks: one of dimension $n-1$ corresponding to an eigenvalue $\lambda,|\lambda|=1$ and the second one-dimensional block corresponding to an eigenvalue $\mu,|\mu|=1$. For $x, y \in H$ we have $\left\langle T^{k} x, y\right\rangle=$ $\lambda^{k} p(k)+a \mu^{k}$ for some polynomial $p, \operatorname{deg} p \leq n-2$ and a number $a \in \mathbb{C}$. Again one can show easily that the set $\left\{\left\langle T^{k} x, y\right\rangle: k \in \mathbb{N}\right\}$ cannot be dense in $\mathbb{C}$. Hence there are no weakly numerically hypercyclic $(2 n-3)$-isometries on $H$.

Theorem 3.4. For $m \geq 2$, there exists a numerically hypercyclic strict $m$-isometry on $\ell^{2}(\mathbb{N})$.

Proof. For $m \geq 2$, no strict $m$-isometry is power bounded [11, Theorem 2]. Also by $\left[1\right.$, Theorem 1], there exist forward weighted shifts on $\ell^{2}(\mathbb{N})$ that are strict $m$ isometries for $m \geq 2$. Since a forward weighted shift on $\ell^{p}(\mathbb{N}), 1<p<\infty$ is numerically hypercyclic if and only if $T$ is not power bounded ([17] \& [21]), we obtain the result.

Since both numerical hypercyclicity and $m$-isometry are properties preserved by unitary equivalence, we have that

Corollary 3.2. Let $H$ be an infinite dimensional separable complex Hilbert space and $m \geq 2$. Then there exists a numerically hypercyclic $m$-isometry on $H$.

Theorem 3.5. There exists a numerically hypercyclic Cesàro bounded strict 3-isometry on $\mathbb{C}^{4}$.

Proof. Let $T$ be the operator considered in the proof of Theorem 3.2

$$
T:=\left(\begin{array}{cccc}
\lambda_{1} & \lambda_{1}-1 & 0 & 0 \\
0 & \lambda_{1} & 0 & 0 \\
0 & 0 & \lambda_{2} & \lambda_{2}-1 \\
0 & 0 & 0 & \lambda_{2}
\end{array}\right)
$$

where $\lambda_{1}, \lambda_{2} \in \mathbb{T}$ are rationally independent. By the proof of Theorem 3.2, it is clear that $T$ is numerically hypercyclic. 
Since both blocks

$$
\left(\begin{array}{cc}
\lambda_{1} & \lambda_{1}-1 \\
0 & \lambda_{1}
\end{array}\right) \quad \text { and } \quad\left(\begin{array}{cc}
\lambda_{2} & \lambda_{2}-1 \\
0 & \lambda_{2}
\end{array}\right)
$$

are Cesàro bounded by Lemma 2.1, it is easy to see that $T$ is Cesàro bounded.

We know that there exist examples of numerically hypercyclic and weakly ergodic 3 -isometries. The following result goes further in this direction.

Theorem 3.6. Any weakly ergodic strict 3-isometry on a Hilbert space is weakly numerically hypercyclic.

Proof. If $T$ is a weakly ergodic strict 3-isometry, then there exists $x$ such that $\frac{T^{n} x}{n}$ is weakly convergent but it is not norm convergent. Indeed for a strict 3 -isometry $T$, there exists $x$ such that $\frac{T^{n} x}{n}$ does not converge to zero in norm.

Then, since $x_{n}=\frac{T^{n} x}{n}$ is weakly convergent but it is not norm convergent, by [21, Lemma 6.1] there is $y \in H$ such that $\left\{n\left\langle x_{n}, y\right\rangle: n \in \mathbb{N}\right\}$ is dense on $\mathbb{C}$. Hence $T$ is weakly numerically hypercyclic.

In particular, the example of a weakly ergodic 3-isometry defined in [3, Section $5.2]$ is weak numerically hypercyclic.

Question 3.1. Do there exist numerically hypercyclic weakly ergodic 3-isometries?

Let $T$ be an $m$-isometry. By part (1) of Lemma 2.2, it is clear that $T$ is not hypercyclic. What can we say about dynamical properties of $T^{*}$ ? In general we can not say nothing. However, the following result gives a positive answer for forward weighted shift operators.

Theorem 3.7. Let $S_{w}$ be a forward weighted shift strict m-isometry on $\ell^{2}(\mathbb{N})$. Then

1. $S_{w}^{*}$ is mixing if and only if $m \geq 2$.

2. $S_{w}^{*}$ is chaotic if and only if $m \geq 3$.

Proof. By [1, Theorem 1], a unilateral weighted forward shift on a Hilbert space is an $m$-isometry if and only if there exists a polynomial $p$ of degree at most $m-1$ such that for any integer $n \geq 1$, we have that $p(n)>0$ and $\left|w_{n}\right|^{2}=\frac{p(n+1)}{p(n)}$. Thus for $m \geq 2, S_{w}^{*}$ satisfies condition ii) of (b) from [15, Theorem 4.8] and $S_{w}^{*}$ is mixing. For $m \geq 3, S_{w}^{*}$ satisfies condition ii) of (c) from [15, Theorem 4.8] and $S_{w}^{*}$ is chaotic.

Notice that, if $S_{w}$ is a unilateral forward weighted shift and a strict $m$-isometry on $\ell^{2}(\mathbb{N})$ with $m \geq 2$, then $S_{w}^{*}$ is hypercyclic operator.

Since there exist bilateral forward weighted shifts which are strict $m$-isometries on $\ell^{2}(\mathbb{Z})$ for odd $m$, then we have 
Theorem 3.8. Let $S_{w}$ be a bilateral forward weighted shift strict $m$-isometry on $\ell^{2}(\mathbb{Z})$ with $m>1$. Then $S_{w}^{*}$ is chaotic.

Proof. By [1, Theorem 19 \& Corollary 20], a bilateral weighted forward shift on a Hilbert space is a strict $m$-isometry if and only if there exists a polynomial $p$ of degree at most $m-1$ such that for any integer $n$, we have $p(n)>0$ and $\left|w_{n}\right|^{2}=\frac{p(n+1)}{p(n)}$ and $m$ is an odd integer. Hence, for $m \geq 3, S_{w}^{*}$ satisfies condition ii) of (c) from [15, Theorem 4.13]. Thus $S_{w}^{*}$ is chaotic.

\section{References}

[1] B. Abdullah and T. Le, The structure of $m$-isometric weighted shift operators, Operators and Matrices, 10 (2016), no 2, 319-334.

[2] J. Agler, M. Stankus, $m$-isometric transformations of Hilbert space. I, Integral Equations Operator Theory, 21 (1995), no 4, 383-429.

[3] A. Aleman and L. Suciu, On ergodic operator means in Banach spaces, Integral Equations Operator Theory, 85 (2016), 259-287.

[4] T. Bermúdez, A. Bonilla and N. Feldman, The convex-cyclic operator, J. Math. Anal and Appl., 434 (2016), 1166-1181.

[5] T. Bermúdez, A. Bonilla, V. Müller and A. Peris, Cesàro bounded operators in Banach spaces. Preprint.

[6] T. Bermúdez, I. Marrero, A. Martinón, On the orbit of an $m$-isometry, Integral Equations Operator Theory, 64 (2009), 487-494.

[7] T. Bermúdez, A. Martinón and E. Negrín, Weighted shift operators which are $m$-isometries, Integral Equations Operator Theory, 68 (2010), no. 3, 301-312.

[8] T. Bermúdez, A. Martinón and J. A. Noda, An isometry plus a nilpotent operator is an $m$-isometry, Applications. J. Math. Anal. Appl., 407 (2013), no. 2, 505-512.

[9] N. C. Bernardes Jr, A. Bonilla, V. Müller and A. Peris, Distributional chaos for linear operators, J. Funct. Anal., 265 (2013), 2143-2163.

[10] N. C. Bernardes Jr, A. Bonilla, A. Peris and X. Wu, Distributional chaos for operators in Banach spaces, J. Math. Anal. Appl., 459 (2018), 797-821.

[11] M. Cho, S. Ôta and K. Tanahashi, Invertible weighted shift operators which are m-isometries, Proc. Amer. Math. Soc., 141 (2013), no. 12, 4241-4247.

[12] N. Dunford, Spectral Theory I. Convergence to projections, Trans. Amer. Math. Soc., 54, (1943),185-217. 
[13] R. Émilion, Mean-bounded operators and mean ergodic theorems, J. Funct. Anal., 61 (1985), no. 1, 1-14.

[14] M. Faghih Ahmadi and K. Hedayatian, Hypercyclicity and supercyclicity of $m$-isometric operators, Rocky Mountain J. of Math., 42 (2012), no 1, 15-23.

[15] K.-G. Grosse-Erdmann and A. Peris, Linear Chaos, Springer, London, 2011.

[16] B. Hou and L. Luo : Some remarks on distributional chaos for bounded linear operators, Turk. J. Math., 39 (2015), 251-258.

[17] S. G. Kim, A. Peris and H. G. Song, Numerically hypercyclic operators, Integral Equations Operator Theory, 72 (2012), no. 3, 393-402.

[18] M. Lin, On the uniform ergodic theorem, Proc. Amer. Math. Soc., 43 (1974), 337-340.

[19] A. Montes-Rodríguez, J. Sánchez-Álvarez and J. Zemánek, Uniform Abel-Kreiss boundedness and the extremal behavior of the Volterra operator, Proc. London Math. Soc., 91 (2005),761-788.

[20] R. Sanders, An isometric bilateral shift that is weakly supercyclic, Integral Equations Operator Theory, 53 (2005), no. 4, 547-552.

[21] S. Shkarin, Numerically hypercyclic operators, arXiv:1302.2483v1

[22] Y. Tomilov and J. Zemánek, A new way of constructing examples in operator ergodic theory, Math. Proc. Cambridge Philos. Soc., 137 (2004), no. 1, 209-225.

T. Bermúdez

Departamento de Análisis Matemático, Universidad de La Laguna, 38271, La Laguna (Tenerife), Spain.

e-mail: tbermude@ull.es

A. Bonilla

Departamento de Análisis Matemático, Universidad de La Laguna, 38271, La Laguna (Tenerife), Spain.

e-mail: abonilla@ull.es

V. Müller

Mathematical Institute, Czech Academy of Sciences, Zitná 25, 11567 Prague 1, Czech Republic.

e-mail: muller@math.cas.cz

A. Peris

Institut Universitari de Matemàtica Pura i Aplicada, Universitat Politècnica de València, Edifici $8 E$, Acces F, 4a planta, 46022 València, Spain.

e-mail: aperis@mat.upv.es 